\title{
Coverage Analysis and Efficient Placement of Drone-BSs in 5G Networks ${ }^{\dagger}$
}

\author{
Mohamed Amine Ouamri ${ }^{1,2,3, *}$, , Marius-Emil Oteşteanu ${ }^{4}$, Gordana Barb ${ }^{4}$ and Cedric Gueguen ${ }^{3}$ \\ 1 Laboratoire D'informatique Médicale, Faculté de Technologie, Département d'ATE, \\ Université de Bejaia, Bejaia 06000, Algeria \\ 2 Faculté de Génie Électrique et D'informatique, Département de Télécommunication, \\ Université de Tizi Ouzou, Tizi Ouzou 15000, Algeria \\ 3 UFR Informatique et Électronique, University of Rennes 1/IRISA, 35000 Rennes, France; \\ cedric.gueguen@irisa.fr \\ 4 Department of Communications, Politehnica University Timisoara, 300006 Timisoara, Romania; \\ marius.otesteanu@upt.ro (M.-E.O.); gordana.barb@student.upt.ro (G.B.) \\ * Correspondence: ouamrimouhamedamine@gmail.com \\ + Presented at the 1st International Conference on Computational Engineering and Intelligent Systems, \\ Online, 10-12 December 2021.
}

check for updates

Citation: Ouamri, M.A.; Oteşteanu, M.-E.; Barb, G.; Gueguen, C. Coverage Analysis and Efficient Placement of Drone-BSs in 5G Networks. Eng. Proc. 2022, 14, 18 https://doi.org/10.3390/ engproc2022014018

Academic Editors: Abdelmadjid Recioui, Hamid Bentarzi and Fatma Zohra Dekhandji

Published: 10 February 2022

Publisher's Note: MDPI stays neutral with regard to jurisdictional claims in published maps and institutional affiliations.

Copyright: (C) 2022 by the authors. Licensee MDPI, Basel, Switzerland. This article is an open access article distributed under the terms and conditions of the Creative Commons Attribution (CC BY) license (https:// creativecommons.org/licenses/by/ $4.0 /)$.

\begin{abstract}
The integration of drones as base stations has shown to be a potential approach for the future mobile communication systems. Hence, this emerging technology is currently being investigated within the 3GPP standardization community with the main objective of improving coverage and capacity in dense urban areas. Nevertheless, in order to provide adequate coverage for users, it is necessary to find the optimal location of the Drone-BS. This work proposes a novel approach for the Drone-BS in 5G communication systems, using the meta-heuristic algorithm. Firstly, we analyse the downlink coverage probability according to SINR by using stochastic geometry. Afterwards, we apply the Grey Wolf Optimizer algorithm in order to find the optimal Drone-BS placement under coverage probability constraint.
\end{abstract}

Keywords: drone-Base Station; coverage; 5G networks; Grey Wolf Optimizer

\section{Introduction}

The chronological progress and exponential growth of cellular networks has led the mobile communications community to suggest the use of drones as a Base Station (BS), with the incorporation of a transceiver, due to its massive potential in mobile communication systems [1]. This approach provides significant improved coverage, enhanced quality of service and increased capacity [2,3]. Furthermore, the high position of the drone-BS encourages Line-of-Sight (LOS) communication to grounds users [4], and consequently the optimal drone-BS deployment is paramount for the proper functioning of the network. On the other hand, with the significant increase in traffic volume, 5G mobile networks will exploit the vast amount of available spectrum in the millimeter wave (mmWave) band [5]. Simultaneously, a drone equipped with a mmWave base station offers considerable throughput. Several research projects are paying more attention to the drone-BS deployment. For instance, authors in [6] study the optimal Unmanned Aerial Vehicles (UAV) placement in order to analyse coverage with fixed UAV altitude. Similarly, in [7], the authors investigate the optimal placement of drone-cells by taking into consideration the backhaul requirements. In addition, the authors propose the robustness solution of drone-BS placement under the user's movement. A heuristic approach based on Particle Swarm Optimization (PSO) is introduced in order to find the optimal 3D location of drone-cells in [8]. The study aims to maximize coverage and limit interference while reducing the drone's altitudes. More recently, in [3], the authors propose a novel 3D UAV-BS placement using exhaustive search (ES) and maximal weighted area algorithms. They maximize coverage under the 
quality-of-service requirement constraint. The efficient UAVs deployment in order to provide coverage is suggested in [4]. The authors study the downlink coverage probability as function of altitude and antenna gain and apply circle packing theory in order to maximize the coverage area. Authors in [9] present UAVs-BS placement based on the location of users. In this paper we investigate the drone-BS placement by assuming random distribution and mmWave Path Loss model. We analyse the downlink coverage probability based on altitude and antenna gain. We apply a Grey Wolf Optimizer (GWO) algorithm [10] in order to find the optimal 3D position that satisfies coverage under interference constraint. The rest of this paper is organized as follows: Section 2 presents the system model adopted for our work, Section 3 describes the downlink coverage probability and Section 4 provides the proposed optimal drone-BSs placement algorithm using GWO. Simulation and numerical results are given in Section 5. Finally, conclusion and future work are discussed in Section 6.

\section{System Model}

In this section we present the system model adopted for our work. We consider an urban scenario where $N$ users are distributed randomly in the area represented by $(x j, j)$. The $M$ drones-BSs are randomly distributed with fixed power transmission. Each drone is defined by its altitude $h$ and location $(x i, i)$. Geometrically, the coverage area of the drone is given by $C$ drone $=(R 2-h 2)$, where $R$ represents the radius of the transmitter drone [11]. Figure 1 depicts a possible scenario of drone-BSs deployment. Additionally, we assume that the propagation environments are both LOS and Non-Line-of-Sight (NLOS). On the other hand, the metric for satisfying quality of service is based on the determination of the Signal-to-Interference Plus Noise Ratio (SINR), which should be above a certain threshold SINR > SINRthr. According to [3], the probability of the mean path loss is given by:

$$
P L\left(h, d_{j i}\right)=P L_{L O S} P_{L O S}+P L_{N L O S} P_{N L O S}
$$

where $P_{L O S}$ represents the probability of the LOS link given by Equation (2) and $P_{N L O S}=$ $1-P_{\text {LOS }}$. As discussed in [12], the path loss between drone-BSs and users for the LOS and NLOS propagation in $d B$ is given by Equation (3).

$$
\begin{gathered}
P_{\text {LOS }}=\frac{1}{1+\operatorname{aexp}\left(-b\left(\left(\frac{180}{\pi}\right) \tan ^{-1}\left(\frac{h}{\sqrt{\left(x_{j}-x_{i}\right)^{2}+\left(y_{j}-y_{i}\right)^{2}}}\right)-a\right)\right)} \\
P L_{L O S}=20 \log \left(\frac{4 \pi f_{c} d_{j i}}{c}\right)+\eta_{L O S} \\
P L_{N L O S}=20 \log \left(\frac{4 \pi f_{c} d_{j i}}{c}\right)+\eta_{N L O S}
\end{gathered}
$$

where $f_{c}$ represents the carrier frequency, $d_{j i}=\sqrt{\left(x_{j}-x_{i}\right)^{2}+\left(y_{j}-y_{i}\right)^{2}+h^{2}}$ is the distance between the drone-BSs and user $i$. Furthermore, $\eta_{L O S}$ and $\eta_{N L O S}$ are the average additional losses for LOS and NLOS, respectively. The probability mean path loss can be further rewritten as:

$$
P L\left(h, d_{j t}\right)=\left(\eta_{L O S}-\eta_{N L O S}\right) P_{L O S}+10 \log \left(\sqrt{\left(x_{j}-x_{i}\right)^{2}+\left(y_{j}-y_{i}\right)^{2}}\right)+B
$$

with $B=20 \log \left(\frac{4 \pi f_{c}}{c}\right)+\eta_{N L O S}$. 


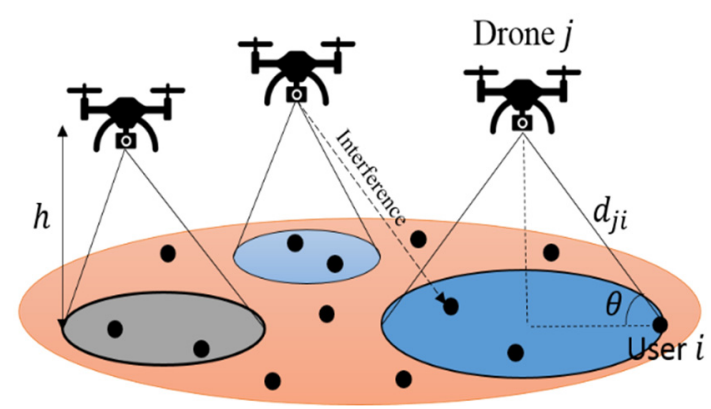

Figure 1. System Model.

As shown in Figure 1, the blue circles represent the drone's coverage area, and the black points indicate the users in the area. At this point, there are two major binary decisions to be conducted. The first one is to determine the deployment of drone-BSs and the second one is to determine the users covered by drone-BSs. The procedure can be developed mathematically as follows: let $D=\{1 \ldots M\}$ represent the set of the candidate drone-BSs and $U=\{1 \ldots N\}$ the set of the users. For the first case, the binary decision can be formulated as:

$$
\omega_{x y h}^{i}=\left\{\begin{array}{l}
1, \text { if the drone }-B S \text { is deployed at }\left(x_{i}, y_{i}, h\right) \\
0, \text { otherwise }
\end{array}\right.
$$

Then, for the second case (user's coverage):

$$
\varphi_{j}^{i}=\left\{\begin{array}{l}
1, \text { if user } j \text { is covered by one drone }-B S i \\
0, \text { otherwise }
\end{array}\right.
$$

\section{Downlink Coverage Probability Calculation}

In this section we study the downlink coverage probability. When evaluating channel performance, a typical user in the area is associated with a drone-BS when the SINR is greater than a predefined threshold. According to [3], the received power at the user $j$ from its drone-BS is given by:

$$
p_{r}^{j i}=p_{t}-P L\left(h, d_{j i}\right)-\vartheta
$$

where $p_{t}$ is the power transmission and $\vartheta$ is the shadow fading with Gaussian random variable in $\mathrm{dB}$ for LOS and NLOS environments. The SINR ratio is given by the following formula:

$$
\begin{gathered}
\text { SINR }=\frac{p_{r}^{j i}}{\sum_{i^{\prime} \in D, i^{\prime} \neq i} p_{r}^{i^{\prime} j}+N} \\
\mathbb{P}\left[S I N R>S I N R_{t h r}\right]=\mathbb{P}\left[\frac{p_{r}^{j i}}{\sum_{i^{\prime} \in D, i^{\prime} \neq i} p_{r}^{j i^{\prime}}+N} \geq S I N R_{t h r}\right]
\end{gathered}
$$

Lemma 1. In the drone-BS network, the downlink coverage probability for a ground user located at a distance $d_{j i}$ from drone-BS can be expressed as:

$$
p_{c}=Q\left(\frac{\left(P L\left(h, d_{j i}\right)+S I N R_{t h r}\left(\sum_{i^{\prime} \in D, i^{\prime} \neq i} p_{r}^{j i^{\prime}}+N\right)-p_{t}+\mu\right)}{\sigma}\right)
$$

where $Q($.$) is the Q$ function. 
Proof.

$$
\begin{gathered}
p_{c}=\mathbb{P}\left[\frac{p_{r}^{j i}}{\sum_{i^{\prime} \in D, i^{\prime} \neq i} p_{r}^{j i^{\prime}}+N} \geq S I N R_{t h r}\right] \\
=\mathbb{P}\left[p_{r}^{j i} \geq \operatorname{SINR} R_{t h r}\left(\sum_{i^{\prime} \in D, i^{\prime} \neq i} p_{r}^{j i^{\prime}}+N\right)\right] \\
=\mathbb{P}\left[p_{t}-P L\left(h, d_{j i}\right)-\vartheta \geq \operatorname{SINR} R_{t h r}\left(\sum_{i^{\prime} \in D, i^{\prime} \neq i} p_{r}^{j i^{\prime}}+N\right)\right] \\
=\mathbb{P}\left[\vartheta \leq p_{t}-P L\left(h, d_{j i}\right)-S I N R_{t h r}\left(\sum_{i^{\prime} \in D, i^{\prime} \neq i} p_{r}^{j i^{\prime}}+N\right)\right] \\
Q\left(\frac{\left(P L\left(h, d_{j i}\right)+S I N R_{t h r}\left(\sum_{i^{\prime} \in D, i^{\prime} \neq i}^{j i^{\prime}}+N\right)-p_{t}+\mu\right)}{\sigma}\right)
\end{gathered}
$$

where $\vartheta \sim N\left(\mu_{L O S}, \sigma_{L O S}^{2}\right)$ and $\vartheta \sim N\left(\mu_{N L O S}, \sigma_{N L O S}^{2}\right)$ represent the shadow fading with normal distribution, in $\mathrm{dB}$, for LOS and NLOS cases, respectively. However, the Complementary Cumulative Distribution Function (CCDF) of a Gaussian random variable as show in Equation (12) below gives the downlink coverage probability for LOS environment.

$$
p_{c}=Q\left(\frac{\left(P L_{L O S} P_{L O S}+S I N R_{t h r}\left(\sum_{i^{\prime} \in D, i^{\prime} \neq i} p_{r}^{j i^{\prime}}+N\right)-p_{t}+\mu_{L O S}\right)}{\sigma_{L O S}}\right)
$$

\section{Optimal Drone-BSs Placement}

In this section we propose to solve the 3D deployment of drone-BSs using GWO [10], where the goal is to maximize coverage by increasing the number of users covered by drone-BSs. Generally, the drone-BSs placement constitutes a NP-hard problem. For this reason, evolutionary algorithms can be a suitable alternative. Mathematically, the coverage problem can be formulated using Mixed Integer Non-Linear Programming (MINLP), as follows:

$$
\max _{x_{i}, y_{i}, h, \varphi_{j}^{i}} \sum_{j=1}^{N} \sum_{i=1}^{M} \varphi_{j}^{i}
$$

Subject to:

$$
\begin{aligned}
& \min \sum_{i=1}^{M} \omega_{x y h}^{i} \\
& h_{\min } \leq h \leq h_{\max } \\
& \sum_{j=1}^{N} b_{j} \varphi_{j}^{i} \leq B W
\end{aligned}
$$

The first constraint assumes that the coverage must be met with a minimum number of drone-BSs deployed. Indeed, a considerable deployment of drones leads to a reduction in the distance between them and hence, the interference from the adjacent drone-BSs increases [4]. The variables $h_{\text {min }}, h_{\text {max }}$ in the second constraint represent the minimum and maximum drone-BS's altitude, respectively. Nevertheless, as the altitude of droneBS increases, the coverage radius also increases, and energy performance of drone-BSs decreases. The last proposed constraint indicates the total limiting available bandwidth, where $b j$ is the bandwidth required by user $j$ and $B W$ denotes the total bandwidth of the drone-BS [7]. Now we apply GWO in order to find the optimal 3D drone-BSs placement. Proposed by [10], GWO is a meta-heuristic algorithm inspired by the Grey Wolf hunting mechanism. The GWO algorithm takes into consideration the search, encircling and attacking prey by Grey Wolf. Notwithstanding, the algorithm classifies wolf into four groups to find leadership: $\alpha$ is the most optimal solution and $\beta$, are the second and 
third best solutions, respectively. $X$ represents the alternative solutions. The process is mathematically modelled as follows:

$$
X(t+1)=\frac{1}{3}\left(X_{1}(t)+X_{2}(t)+X_{3}(t)\right)
$$

where:

$$
\begin{aligned}
& X_{1}(t)=X_{\alpha}-A_{1} \times D_{\alpha} \\
& X_{2}(t)=X_{\beta}-A_{2} \times D_{\beta} \\
& X_{3}(t)=X_{\theta}-A_{3} \times D_{\theta}
\end{aligned}
$$

From the equations above, $X_{1}(t), X_{2}(t), X_{3}(t)$ are the position vectors of the prey, and $X(t+1)$ represents the grey wolf position vector. Grey wolves encircle prey during the hunt. The mathematically model-encircling behaviour is given by the following formula:

$$
D=\left|C \times X_{p}(t)-X(t)\right|
$$

where: $C=2 \times r_{2}$ and $A=2 a \times r_{2}-a$ are two coefficient vectors, and $r_{1}, r_{2}$ are vectors randomly generated between $[0,1]$. The Algorithm 1 of GWO is given by:

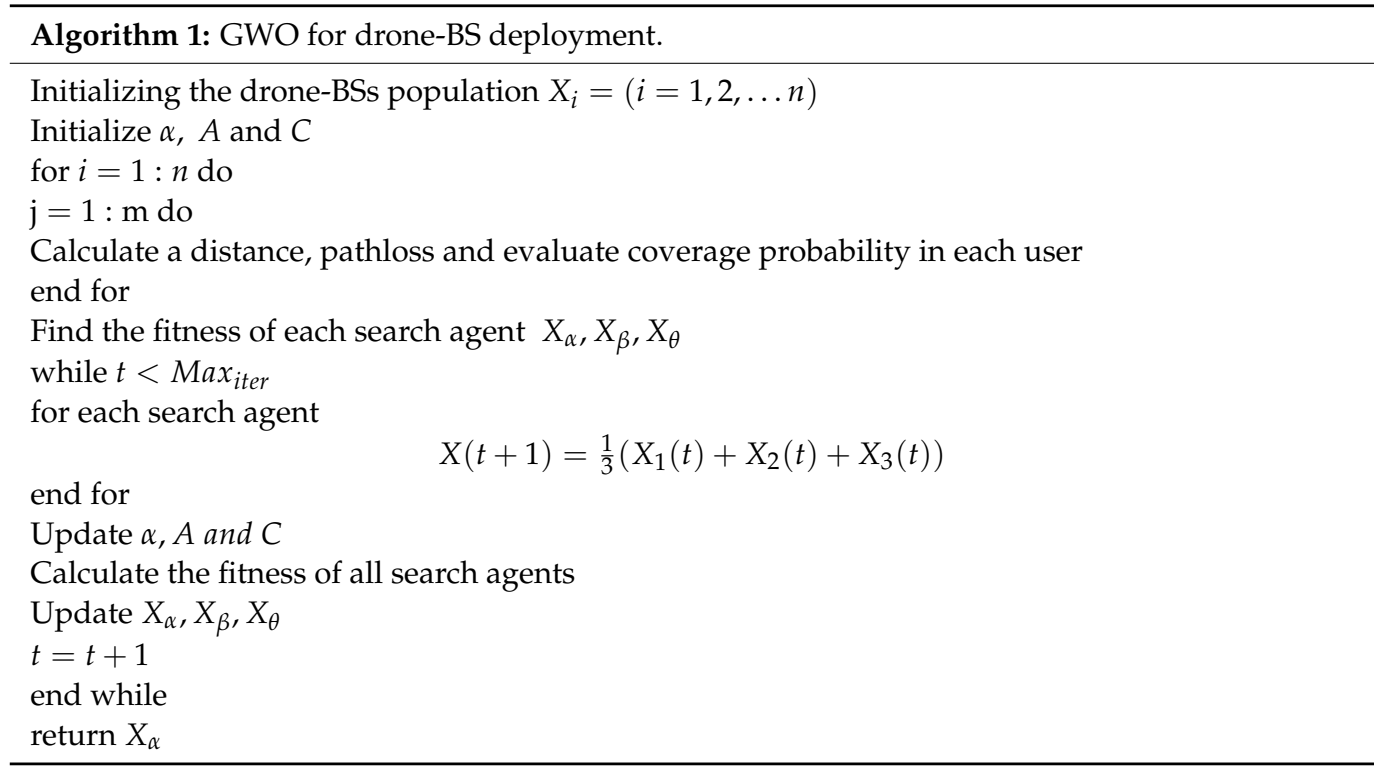

\section{Simulation Results}

The simulation and numerical results for the downlink coverage probability and optimal drone-BSs placement are presented in this section. 200 users and 10 drone-BSs are randomly distributed within a $2 \times 2 \mathrm{~km}^{2}$ area. The simulation parameters are illustrated in Table 1. Our goal is to serve the maximum number of users based on a reduced number of drone-BSs using a mmWave carrier frequency.

Table 1. Simulation parameters adopted for our work.

\begin{tabular}{cc}
\hline Parameters & Value \\
\hline$f_{c}$ & $28 \mathrm{GHz}$ \\
$h_{\min } \leq h \leq h_{\max }$ & $1000 \leq h \leq 3000 \mathrm{~m}$ \\
$M$ (number of drone-BSs) & 10 \\
$N$ (number of users) & 200 \\
$p_{t}$ & $30 \mathrm{dBm}$ \\
$S I N R_{t h r}$ & $5 \mathrm{dBm}$ \\
$B$ & $20 \mathrm{MHz}$ \\
\hline
\end{tabular}


Figure 2 depicts the downlink coverage probability considering different altitudes for the drone-BSs. We observe that the coverage probability increases when the altitude of the drone-BSs also increases. However, as mentioned above, a very high altitude can influence the energy performance of the drone. Figure 3 shows the optimal drone-BSs placement and the minimum required number of drone-BSs to ensure that all users are covered. The results indicate that in order to satisfy 0.76 of downlink coverage probability, it is necessary to deploy five drone-BSs. On the other hand, a coverage probability can be improved by increasing the number of drones, although this would lead to a decrease in the distance between the drones and consequently the interference at user level would be higher.

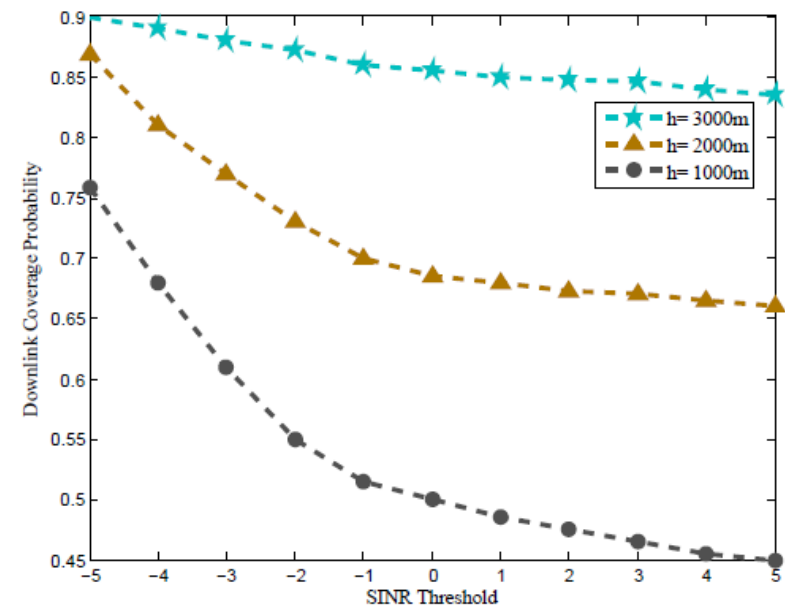

Figure 2. Coverage probability versus SINR threshold, at different altitudes.

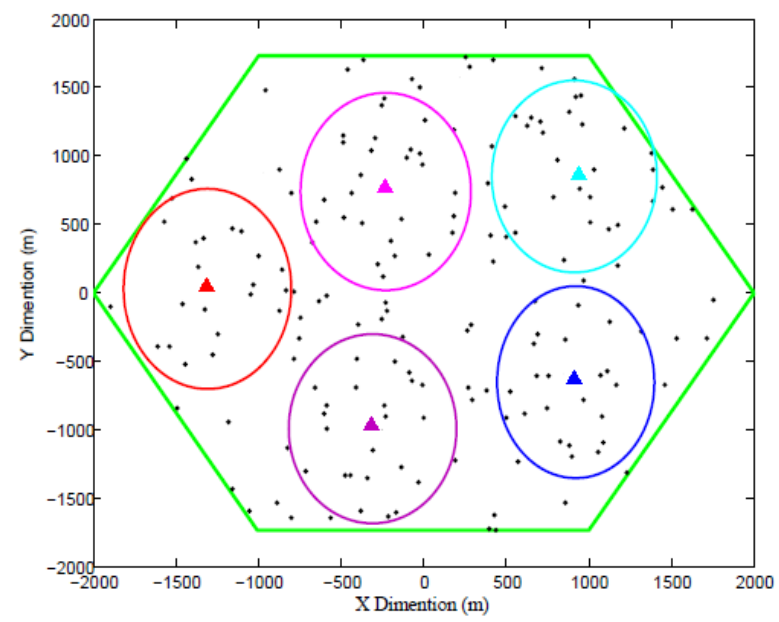

Figure 3. Drone-BSs placement at the end of the GWO iteration.

Furthermore, the number of users served by the deployed drone-BSs are illustrated in Figure 4. We observe a decrease in the number of users covered by the network when the number of drone-BSs is reduced. Moreover, the results obtained indicate that the drone-BSs are separated in distance, leaving uncovered areas. Besides, the optimal altitude selected for the drone-BSs can be improved to increase the coverage radius and get more users covered, but with reduced energy performance. 


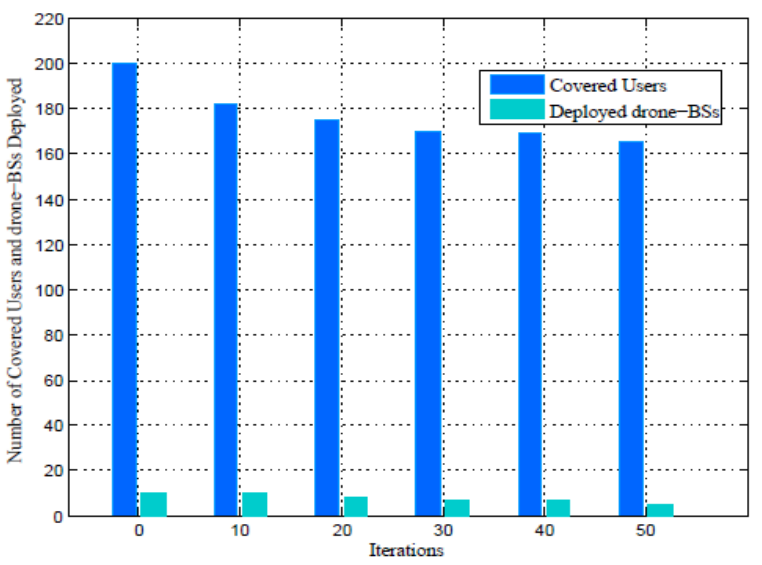

Figure 4. Number of covered users and drone-BSs deployment at each iteration.

\section{Conclusions}

Drone-BSs deployment in 5G networks is currently an important subject with extreme potential in the mobile communication networks industry. Placement optimization is a complicated task that requires automation algorithms to find the optimal solution for the placement of drone-BSs. In this work, we studied a 3D deployment of drone-BSs in a 5G network using GWO algorithm. Firstly, we analysed the downlink coverage probability according to SINR and altitude. Next, we proposed a mathematical model in order to maximize coverage under a minimum number of drone-BSs, altitude and total limiting available bandwidth. The results obtained showed a minimization of the total number of drone-BSs deployed with a coverage reaching $82.5 \%$. Our study does not take into consideration the blocking effect and overlap between drones that are important for the handoff, but these factors will be the focus of research in future work.

Author Contributions: Conceptualization, M.A.O. and G.B.; methodology, C.G.; software, M.A.O.; validation, M.-E.O. and C.G.; formal analysis, M.A.O.; investigation, G.B.; resources, G.B.; writingreview and editing, C.G. and M.-E.O.; supervision, M.-E.O.; All authors have read and agreed to the published version of the manuscript.

Funding: This Project is financed by Politehnica University Timisoara, Timisoara 300006, Romania.

Institutional Review Board Statement: Not applicable.

Informed Consent Statement: Not applicable.

Data Availability Statement: Not applicable.

Acknowledgments: The authors wish to acknowledge the technical support, infrastructure and documentation provided by Nokia Networks.

Conflicts of Interest: The authors declare no conflict of interest.

\section{References}

1. Bor-Yaliniz, I.; Yanikomeroglu, H. The New Frontier in RAN Heterogeneity: Multi-Tier Drone-Cells. IEEE Commun. Mag. 2016, 54, 48-55. [CrossRef]

2. Mozaffari, M.; Kasgari, A.T.Z.; Saad, W.; Bennis, M.; Debbah, M. Beyond 5G With UAVs: Foundations of a 3D Wireless Cellular Network. IEEE Trans. Wirel. Commun. 2019, 18, 357-372. [CrossRef]

3. Alzenad, M.; El-Keyi, A.; Yanikomeroglu, H. 3-D Placement of an Unmanned Aerial Vehicle Base Station for Maximum Coverage of Users with Different QoS Requirements. IEEE Wirel. Commun. Lett. 2018, 7, 38-41. [CrossRef]

4. Mozaffari, M.; Saad, W.; Bennis, M.; Debbah, M. Efficient Deployment of Multiple Unmanned Aerial Vehicles for Optimal Wireless Coverage. IEEE Commun. Lett. 2016, 20, 1647-1650. [CrossRef]

5. Chen, S.; Zhao, J. The requirements, challenges, and technologies for $5 \mathrm{G}$ of terrestrial mobile telecommunication. IEEE Commun. Mag. 2014, 52, 36-43. [CrossRef] 
6. Li, X.; Guo, D.; Yin, H.; Wei, G. Drone-assisted public safety wireless broadband network. In Proceedings of the 2015 IEEE Wireless Communications and Networking Conference Workshops (WCNCW), New Orleans, LA, USA, 9-12 March 2015; pp. 323-328.

7. Kalantari, E.; Shakir, M.Z.; Yanikomeroglu, H.; Yongacoglu, A. Backhaul-aware robust 3D drone placement in 5G+ wireless networks. In Proceedings of the 2017 IEEE International Conference on Communications Workshops (ICC Workshops), Paris, France, 21-23 May 2017; pp. 109-114.

8. Kalantari, E.; Yanikomeroglu, H.; Yongacoglu, A. On the Number and 3D Placement of Drone Base Stations in Wireless Cellular Networks. In Proceedings of the 2016 IEEE 84th Vehicular Technology Conference (VTC-Fall), Montreal, QC, Canada, 18-21 September 2016; pp. 1-6.

9. Bor-Yaliniz, R.I.; El-Keyi, A.; Yanikomeroglu, H. Efficient 3-D placement of an aerial base station in next generation cellular networks. In Proceedings of the 2016 IEEE International Conference on Communications (ICC), Kuala Lumpur, Malaysia, 23-27 May 2016; pp. 1-5.

10. Mirjalili, S.; Mirjalili, S.M.; Lewis, A. Grey Wolf Optimizer. Elsevier Adv. Eng. Softw. 2014, 69, 46-61. [CrossRef]

11. Al-Turjman, F.; Lemayian, J.P.; Alturjman, S.; Mostarda, L. Enhanced Deployment Strategy for the 5G Drone-BS Using Artificial Intelligence. IEEE Access 2019, 7, 75999-76008. [CrossRef]

12. Al-Hourani, A.; Kandeepan, S.; Lardner, S. Optimal LAP Altitude for Maximum Coverage. IEEE Wirel. Commun. Lett. 2014, 3, 569-572. [CrossRef] 